\title{
Resveratrol provides benefits in mice with type II diabetes-induced chronic renal failure through AMPK signaling pathway
}

\author{
HAIYAN GUO $^{1}$ and LINYUN ZHANG ${ }^{2}$ \\ ${ }^{1}$ Department of Clinical Medicine, Fenyang College, Shanxi Medical University; ${ }^{2}$ Department of Internal Medicine, \\ Shanxi Fenyang Prison Hospital, Fenyang, Shanxi 032200, P.R. China
}

Received August 7, 2016; Accepted May 11, 2017

DOI: $10.3892 /$ etm.2018.6178

\begin{abstract}
Type II diabetes-induced ischemic injuries are known to lead to the rapid degeneration of the kidneys as a result of chronic renal failure. Chronic renal failure is a condition, which typically manifests with symptoms including cardiovascular system and left ventricular hypertrophy, atherosclerosis as well as arterial and aortic stiffness. Resveratrol is a multifunctional compound that has been reported to produce beneficial outcomes for patients with type-II diabetes due to prevention of oxidative stress and apoptosis. However, the beneficial effects of resveratrol in chronic renal failure and the underlying mechanisms have remained to be fully elucidated. The present study investigated the therapeutic effects of resveratrol in mice with chronic renal failure induced by type-II diabetes and assessed the mechanism of action. Oxidative stress, apoptosis and adenosine monophosphate-activated protein kinase (AMPK) in the renal cells of the model mice were assessed. Changes in inflammatory factors renal cells from experimental mice as well as insulin resistance were also analyzed. Morphological changes and immunocytes in renal cells were determined by immunostaining. The results demonstrated that resveratrol treatment decreased the apoptotic rate of renal cells from experimental mice. Oxidative stress also improved in renal cells, as indicated by inhibition of superoxide dismutase and reduced glutathione and 4-hydroxy-2-nonenal levels. In addition, insulin resistance was improved after an 8 -week treatment with resveratrol. Inflammatory factors were decreased and factors promoting kidney function were increased after resveratrol treatment. Furthermore, morphological changes were observed to be ameliorated, indicating the therapeutic efficacy of resveratrol. In addition, immunocyte precipitation in renal cells was markedly decreased in resveratrol-treated mice. Importantly,
\end{abstract}

Correspondence to: Professor Haiyan Guo, Department of Clinical Medicine, Fenyang College, Shanxi Medical University, 16 Yingxiongbei Road, Fenyang, Shanxi 032200, P.R. China E-mail: guohaiyanmedicine@yeah.net

Key words: type II diabetes, chronic renal failure, inflammation, resveratrol, AMPK the AMPK signaling pathway was found to be involved in the beneficial effect of resveratrol on the model mice. In conclusion, the present study suggested that resveratrol may be an ideal agent for the treatment of chronic renal failure induced by type-II diabetes through regulation of the AMPK signaling pathway, which should be further investigated in clinical trials.

\section{Introduction}

Chronic renal failure is a systemic urological condition caused by a variety of chronic diseases and progressive deterioration leading to kidney damage that represents a global public health problem (1). In recent years, chronic renal failure has had a significantly increasing trend and studies have highlighted the role of type II diabetes in the initiation and progression of chronic kidney disease $(2,3)$. In addition, patients with cancers frequently presented with acute renal failure and membranoproliferative glomerulonephritis after radiotherapy and chemotherapy (4). Studies have suggested that chronic systemic inflammation contributes to the progression of chronic renal failure and is associated with complications, including heart disease, cardiovascular disease and metabolic disorders $(5,6)$. Evidence has indicated that type II diabetes-induced inflammatory responses promote the initiation and development of chronic renal failure $(7,8)$. Therefore, exploring efficient methods to solve chronic renal failure induced by type II diabetes is urgently required for patients in the clinic.

Inflammation is one of the most common characteristics of in patients with Type II diabetes (9), and is associated with the dysfunction of urinary albumin excretion, endothelial function and cellular metabolism (10). Therefore, hypertension is also common in patients with type-II diabetes and its onset is frequently discovered in clinical investigations (11-13). A previous study has reported that inhibition of the renin-angiotensin system exerted potent effects in decreasing blood pressure by reducing vascular inflammation (14). However, long-term medication of anti-hypertensive drug leads to decline of renal function and even causes chronic renal failure in patients with type II diabetes (15). Chronic renal failure is a syndrome associated with serious metabolic disorders caused by a variety of chronic kidney diseases (16). The major conditions associated with its pathogenesis are glomerulonephritis, interstitial nephritis, high blood pressure, diabetes and obstructed kidney disease (17). With the rapid 
increase of diabetes patients worldwide, diabetes-associated chronic renal failure has recently demonstrated an increasing trend in clinical investigations $(18,19)$.

Resveratrol is a multifunctional compound that has been reported to provide beneficial outcomes for patients with type-II diabetes due to the prevention of oxidative stress and apoptosis (20-22). Resveratrol is a naturally existing polyphenol, which has provided therapeutic effects in the treatment of diabetes and gestational diabetes mellitus (GDM) in most of the available previous studies $(23,24)$. In addition, resveratrol has been found to be beneficial in the treatment of human diseases, such as cancer, neurodegenerative diseases, cerebrovascular conditions, type II diabetes mellitus and GDM $(25,26)$. Furthermore, high glucose-induced cardiomyocyte apoptosis and oxidative stress have been proved to be associated with the adenosine monophosphate-activated protein kinase (AMPK) signaling pathway (27). However, the effects of resveratrol on chronic renal failure remained to be fully elucidated. The present study hypothesized that improvement of chronic renal failure after resveratrol treatment may be regulated through the AMPK pathway.

In the present study, the influence of resveratrol on chronic renal failure induced by type II diabetes was investigated. The activation of oxidative stress, apoptosis and inflammatory factors was analyzed in the renal cells from experimental mice with chronic renal failure induced by type II diabetes after treatment with resveratrol. The results demonstrated that resveratrol regulated inflammatory responses and improved morphological changes and immunocytes in renal tissues through the AMPK signaling pathway. These findings suggested that resveratrol targeting the mitochondrial AMPK signaling may be a promising strategy to improve chronic renal failure induced by type II diabetes.

\section{Materials and methods}

Ethics statement. This pre-clinical study was performed according to the recommendations in the Guide for the Care and Use of Laboratory Animals of Fenyang College Shanxi Medical University (Fenyang, China). All experimental protocols on animals were in accordance with the Guide for the Care and Use of Laboratory Animals of the National Institutes of Health and approved by the Ethics Committee of Fenyang College Shanxi Medical University. All surgeries and euthanasia were performed in a manner of causing minimal suffering.

Animals and study design. A total of $60 \mathrm{C} 57 \mathrm{BL} / \mathrm{KsJ} \mathrm{db} /+(\mathrm{db} /+)$ mice with type II diabetes (age, 6-8 weeks) were purchased from Charles River Laboratories (Sulzfeld, Germany). All mice were housed in a temperature-controlled room $\left(25 \pm 1^{\circ} \mathrm{C}\right)$ with a 12-h light/dark cycle. The mice received insulin once a day for a 365-day observation. The concentration of creatinine in the serum was analyzed to identify those mice in which chronic renal failure was induced by type II diabetes. The mice with chronic renal failure induced by type II diabetes were divided into three groups $(\mathrm{n}=20$ in each) and received resveratrol (10 mg/kg; Sigma-Aldrich; Merck KGaA, Darmstadt, Germany), angiotensin-converting enzyme inhibitor (ACEI) aldosterone (10 mg/kg, Sigma-Aldrich; Merck KGaA,
Darmstadt, Germany) or PBS by intravenous injection, and 20 healthy C57BL/KsJ+/+ (wild-type) mice (6-8 weeks old; Beijing University, Beijing, China) were used as controls. The treatments were continued for 8 weeks at one-day intervals. The renal cells were isolated from experimental mice for further analysis on day 60 .

Glucose and insulin tolerance tests. Mice with chronic renal failure induced by type II diabetes were fasted $6 \mathrm{~h}$ and injected intraperitoneally with glucose at a dose of $2 \mathrm{~g} / \mathrm{kg}$ for the glucose tolerance test. Blood glucose concentrations were analyzed using an ACCU-CHEK Advantage glucometer (Roche Diagnostics, Basel, Switzerland). The results of the glucose tolerance tests were recorded at baseline and after glucose injection (120 min). For the insulin tolerance tests, mice with GDM insulin were injected with insulin intraperitoneally at $0.75 \mathrm{U} / \mathrm{kg}$ body weight. The blood glucose concentration was measured at baseline and $30 \mathrm{~min}$ after GDM mice received resveratrol, aldosterone or PBS.

Physical activity analysis by indirect calorimetry. Indirect calorimetric analysis was used to evaluate the physical activity of mice with chronic renal failure induced by type II diabetes by using the Comprehensive Laboratory Animal Monitoring System (Oxymax/CLAMS; Columbus Instruments Corp., Columbus, OH, USA). On day 60 after treatment with resveratrol, ACEI or PBS, physical activities of mice were monitored every $30 \mathrm{~min}$ for $24 \mathrm{~h}$ to analyze the therapeutic effects of the treatments on chronic renal failure induced by type II diabetes. The respiratory exchange ratio, volume of oxygen $\left(\mathrm{VO}_{2}\right)$, $\mathrm{VCO}_{2}$, heat production and physical activity were measured according to the manufacturers' instructions.

Glucose-6-phosphatase activity. Glucose-6-phosphatase activity of mice with chronic renal failure induced by type II diabetes was analyzed as previously described (28). Total protein in the blood of mice with chronic renal failure induced by type II diabetes was analyzed by the Bradford method. Glucose-6-phosphatase activity was normalized by subtracting nonspecific phosphatase activity determined by para-nitrophenylphosphate (p-NPPSt, Sigma-Aldrich; Merck $\mathrm{KGaA})$.

Analysis of apoptosis. Renal sections from experimental mice were prepared for apoptotic analysis. Renal cells were incubated with terminal deoxynucleotidyl transferase deoxyuridine triphosphate nick end labeling (TUNEL) stain (Invitrogen; Thermo Fisher Scientific, Inc., Waltham, MA, USA) for $60 \mathrm{~h}$ according to the protocol of previous study (29). The apoptotic renal cells were analyzed by fluorescence microscopy as described in a previous study (30).

ELISA. ELISA kits (Huiying, Shanghai, China) were used to determine interleukin (IL)-6 (HY1738), IL-10 (HY1798), IL-17 (HY2013), IL-1 $\beta$ (HY0028), vascular endothelial growth factor (VEGF, HY3014) and tumor necrosis factor (TNF)- $\alpha$ (HY62203) levels in the sera of the mice. The procedures were performed according to the manufacturer's instructions. The final results were recorded at $450 \mathrm{~nm}$ on an ELISA plate reader. 
Table I. Primer sequences of inflammation-associated cytokines used for polymerase chain reaction in the present study.

\begin{tabular}{lll}
\hline Target gene & \multicolumn{1}{c}{ Forward primer } & \multicolumn{1}{c}{ Reverse primer } \\
\hline Caspase-3 & 5'-GTCCCACT-GTCTGTCTCA-3' & 5'-GAATGTCATCTCCGCTCTG-3' \\
Caspase-8 & 5'-AGACATAACCCAACTCCG-3' & 5'-TCATCAGGCACTCCTTTC-3' \\
Caspase-9 & 5'-AATCCTGCTTGGGTATCAGG-3' & 5'-GAGACCCAGTCTCAGGGAAA-3' \\
Apaf-1 & 5'-ACTTGTCGGCCCTGCGCATC-3' & 5'-GGGGCGAACGACTAAGCGGG-3' \\
$\beta$-actin & 5'-GTGGGCGCCCAGGCACCA-3' & 5'-CTCCTTAATGTCACGCACGATTT-3'
\end{tabular}

Apaf-1, apoptotic protease activating factor 1 .

Cell viability assay. Renal cells from experimental mice $\left(1.0 \times 10^{3}\right)$ were seeded in 96-well plates in MEM with $10 \%$ FBS in a final volume of $100 \mu \mathrm{l} /$ well at $37^{\circ} \mathrm{C}$. Following $24 \mathrm{~h}$, cell viability was evaluated using the MTS assay (CellTiter $96^{\circledR}$ AQueous One Solution Cell Proliferation Assay; Promega Corporation) according to the manufacturer's instructions. Cells morphology was observed using a confocal microscope (Carl Zeiss, Zen 2010) (magnification, x40). The final absorbance was recorded using a microplate reader (BMG Labtech Ltd., Aylesbury, UK) at a wavelength of $450 \mathrm{~nm}$. Cells viability was determined by the absorbance of cells at $450 \mathrm{~nm}$.

Reverse-transcription quantitative polymerase chain reaction $(R T-q P C R)$. Total mRNA was isolated from renal cells by using an RNA Easy Mini Extract kit (Sigma-Aldrich; Merck KGaA). RNA was reversed transcribed using a PrimeScript RT Master Mix kit (Takara Bio, Inc., Otsu, Japan). The expression of caspase-3, -8 and -9 as well as apoptotic protease activating factor 1 (Apaf-1) in renal cells was determined by using an RT-qPCR kit (Invitrogen; Thermo Fisher Scientific, Inc.) according to the manufacturer's instructions with $\beta$-actin expression as an endogenous control. All procedures were performed according to the manufacturer's instructions. All of the primers were from Invitrogen (Thermo Fisher Scientific, Inc.) and their sequences are listed in Table I. Relative mRNA expression levels were determined by using the $2^{-\Delta \Delta \mathrm{Cq}}$ method (31). The final results were presented as the fold of $\beta$-actin.

Western blot analysis. Renal tissues were isolated from mice with chronic renal failure induced by type II diabetes and homogenized in $1 \mathrm{X}$ radioimmunoprecipitation assay buffer on day 60. Subsequently, western blotting was performed to analyze the expression of analyte proteins. Protein concentrations were examined using a BCA protein assay, and protein samples $(40 \mu \mathrm{g})$ were loaded and separated using $15 \%$ SDS-PAGE. Protein were subsequently blotted on a nitrocellulose membrane and hybridized using primary antibodies against AMPK $(1: 2,000 ; 9839)$, phosphorylated AMPK $(1: 2,000 ; 4186)$, and $\beta$-actin $(1: 500 ; 3700 ;$ Cell Signaling Technologies, Inc., Danvers, MA, USA) were added for $12 \mathrm{~h}$ at $4^{\circ} \mathrm{C}$ after blocking with $5 \%$ skimmed milk for $1 \mathrm{~h}$ at $37^{\circ} \mathrm{C}$, and membranes were then incubated with horseradish peroxidase-conjugated goat anti-rabbit $\operatorname{IgG} \mathrm{mAb}(1: 5,000$; PV-6001; ZSGB-BIO, Beijing, China) for $24 \mathrm{~h}$ at $4^{\circ} \mathrm{C}$. The blots were visualized by using a chemiluminescence detection system (32209; Invitrogen; Thermo Fisher Scientific, Inc.). Densitometric quantification of the immunoblot data was performed by using the software of Quantity-One (version 3.2; Bio-Rad Laboratories, Inc., Hercules, CA, USA).

Immunohistochemical staining. Immunohistochemical staining was performed by an avidin-biotin-peroxidase technique. Paraffin-embedded tissue sections were prepared and epitope retrieval was performed for further analysis. The paraffin sections were incubated with hydrogen peroxide (3\%) for $15 \mathrm{~min}$ at $37^{\circ} \mathrm{C}$ and subsequently blocked with $5 \%$ skimmed milk for $15 \mathrm{~min}$ at $37^{\circ} \mathrm{C}$. Finally, the sections were incubated in caspase-3 (1:2,000; 9662; Cell Signaling Technologies, Inc.) at $4^{\circ} \mathrm{C}$ for $12 \mathrm{~h}$. All sections were washed 3 times and incubated with horseradish peroxidase-conjugated secondary antibodies (1:2,000; 12768; Cell Signaling Technologies, Inc.) for $1 \mathrm{~h}$ at $37^{\circ} \mathrm{C}$. A total of 6 randomly selected fields of view were observed under the fluorescence microscope (ECLIPSE TE300; Nikon Corporation; Tokyo, Japan). Tissue sections were also stained with 4-hydroxy-2-nonenal (HNE; Cell Signaling Technologies, Inc.) for $2 \mathrm{~h}$ at $37^{\circ} \mathrm{C}$ and measured using ELISA with commercially available kits (MBS027502; MyBioSource; San Diego, CA, USA).

Oxidative stress and anti-oxidant enzyme levels. Renal tissues and cells were isolated from experimental mice and homogenized in buffer containing $1 \mathrm{mM}$ EDTA, $0.5 \mathrm{mM}$ phenylmethane sulfonyl fluoride, $100 \mathrm{mM}$ Tris- $\mathrm{HCl}(\mathrm{pH} 7.4)$ and $0.05 \%$ Triton $\mathrm{X}-100$ with sonication. The homogenates were centrifuged at $8,000 \mathrm{x} \mathrm{g}$ for $10 \mathrm{~min}$ at $4^{\circ} \mathrm{C}$ and the supernatants were used to measure the total thiol, reduced glutathione (GSH), lipid peroxidation and superoxide dismutase (SOD) activity. Total thiol, GSH, lipid peroxidation and superoxide dismutase and reactive oxygen species (ROS) activity in tissue homogenates was evaluated according to a previous study (32). All of the spectrophotometric data were recorded by a Spectramax spectrophotometer (Molecular Devices, Inc., Sunnyvale, CA, USA).

Statistical analysis. Values are expressed as the mean \pm standard deviation from three independent experiments. All data were analyzed using SPSS Statistics 19.0 (IBM Corp., Armonk, NY, USA). Statistical significance of differences between groups was analyzed using a two-tailed Student's t-test. Unpaired data were analyzed by analysis of variance. $\mathrm{P}<0.05$ was considered to indicate a statistically significant difference. 
A

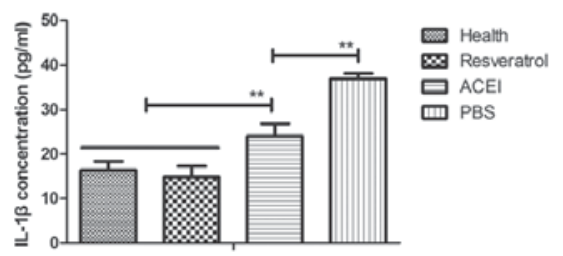

D

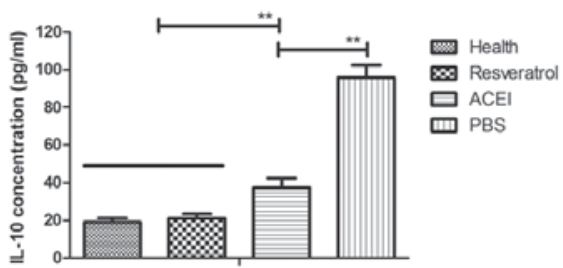

B

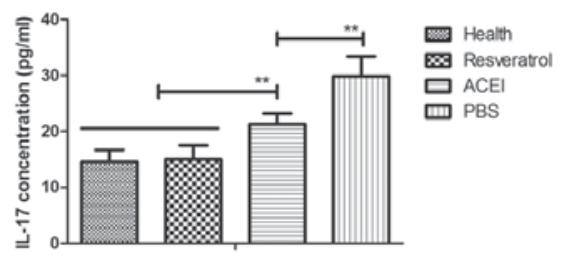

E

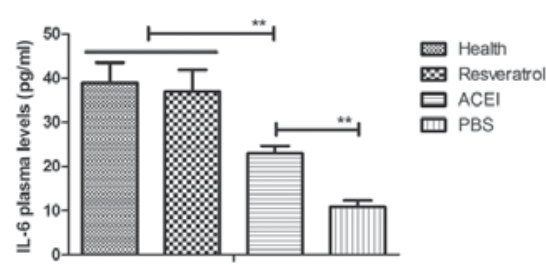

C

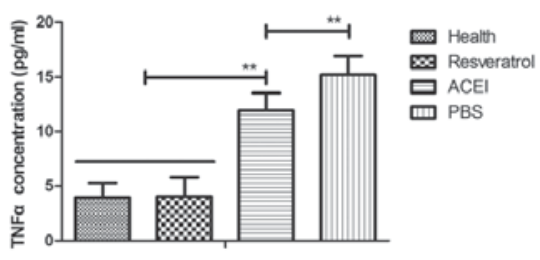

$\mathbf{F}$

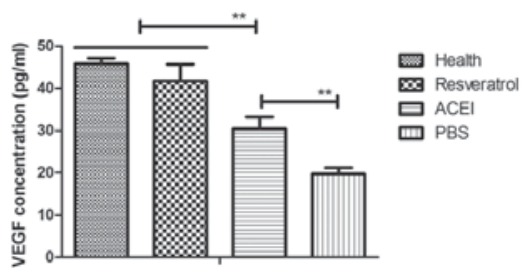

Figure 1. Effects of resveratrol on inflammation in mice with renal failure induced by type II diabetes. Serum levels of (A) IL-1 $\beta$, (B) IL-17, (C) TNF $\alpha$, (D) IL-10 (E) IL-6 and (F) VEGF in the renal cells of experimental mice. Mice with renal failure induced by type II diabetes were treated with resveratrol $(10 \mathrm{mg} / \mathrm{kg})$, the ACEI aldosterone $(10 \mathrm{mg} / \mathrm{kg})$ or the same volume of PBS, and healthy animals were used as controls. ${ }^{* *} \mathrm{P}<0.01$. IL, interleukin; TNF, tumor necrosis factor; VEGF, vascular endothelial growth factor; ACEI, angiotensin-converting enzyme inhibitor.

\section{Results}

Resveratrol improves chronic renal failure induced by type II diabetes by regulation of the inflammatory response. The inflammatory response is crucial for renal failure induced by type II diabetes. Therefore, the present study first analyzed the inflammatory factors in the renal cells from the experimental mice. It was observed that inflammatory factors IL-1 $\beta$, IL-17, IL-10 and TNF- $\alpha$ in the serum in mice with chronic renal failure induced by type II diabetes were significantly increased compared with those in healthy mice, while IL-6 and VEGF were decreased (Fig. 1). However, in comparison with those in the PBS group, the plasma concentrations of IL-1 $\beta$, IL-17, IL-10 and TNF- $\alpha$ were significantly decreased after resveratrol treatment. Furthermore, resveratrol treatment led to increased IL-6 and VEGF levels compared with those in the PBS group. ACEI treatment also significantly decreased IL-1 $\beta$, IL-17, IL-10 and TNF- $\alpha$ and increased IL- 6 and VEGF levels compared with those in the PBS group, but the effect was significantly lower than that of resveratrol. These results indicated that resveratrol regulates inflammatory factors, which may be beneficial for mice with chronic renal failure induced by type II diabetes.

Resveratrol improves glucose metabolism and insulin tolerance in mice with chronic renal failure induced by type II diabetes. To investigate the efficacy of resveratrol on chronic renal failure induced by type II diabetes, its effect on glucose metabolism and insulin tolerance in the experimental mice was assessed. First, changes in the body weight of mice with chronic renal failure induced by type II diabetes were analyzed. The results revealed that in the PBS group, the body weight was significantly decreased compared with that in the Control group, which was inhibited by resveratrol and, to a significantly lesser extent, by ACEI (Fig. 2A). In addition, resveratrol significantly improved the fasting blood glucose concentration in mice with chronic renal failure induced by type II diabetes (Fig. 2B). Resveratrol treatment also significantly alleviated glucose intolerance after the initial glucose injection compared with that in the PBS and ACEI groups (Fig. 2C). Furthermore, resveratrol treatment decreased food intake of mice with chronic renal failure induced by type II diabetes compared with that in the ACEI and PBS groups (Fig. 2D). Of note, insulin levels in mice treated with resveratrol were significantly higher than those in the ACEI and PBS-treated groups (Fig. 2E). Furthermore, the insulin tolerance test indicated that resveratrol treatment significantly improved insulin tolerance in mice with chronic renal failure induced by type II diabetes (Fig. 2F). These results suggested that resveratrol improves body weight, fasting blood glucose concentration and insulin tolerance in mice with chronic renal failure induced by type II diabetes.

Resveratrol reduces renal apoptosis in mice with chronic renal failure induced by type II diabetes. Apoptosis has an important role in mammals with chronic renal failure induced by type II diabetes (33). In the present study, the inhibitory effects of resveratrol on renal cell apoptosis in experimental mice with chronic renal failure were assessed. A TUNEL staining assay revealed that apoptotic renal cells were markedly decreased in mice treated with resveratrol compared with those in the PBS- and ACEI-treated groups (Fig. 3A). Furthermore, resveratrol treatment markedly inhibited the expression of apoptosis-associated genes (Apaf-1, caspase-3, caspase-8 and caspase-9) in renal cells with chronic renal failure (Fig. 3B-E). Immunohistochemical analysis demonstrated that resveratrol treatment significantly decreased caspase- 3 expression, which confirmed the protective effect of resveratrol on renal cells from experimental mice (Fig. 3F). Collectively, these results suggested that resveratrol treatment inhibited apoptosis of renal cells by inactivation of caspase-3 in mice with chronic renal failure induced by type II diabetes. 
A

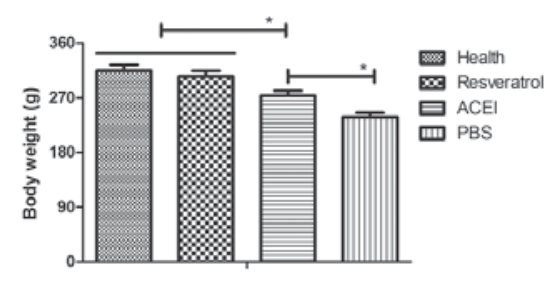

D

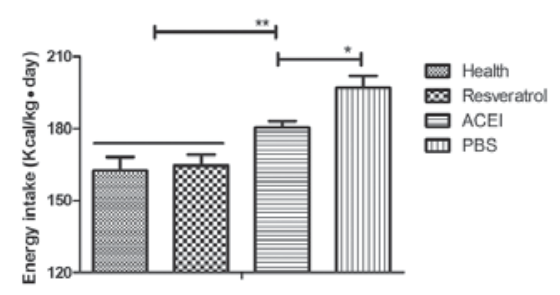

B

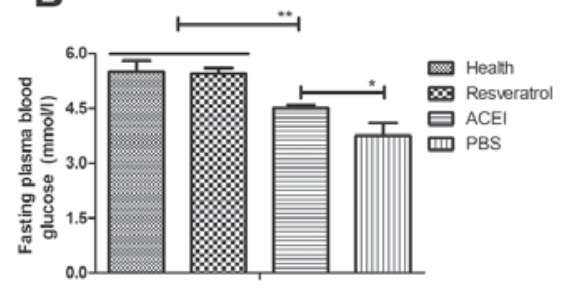

E

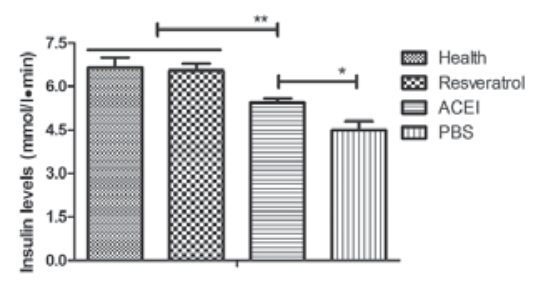

C

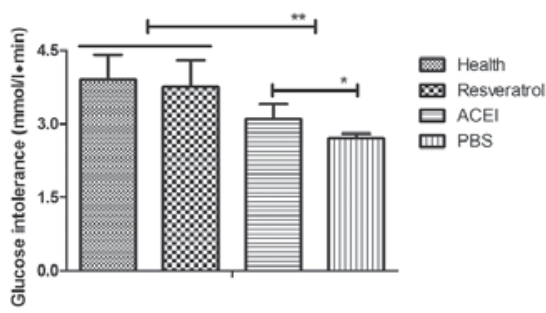

$\mathbf{F}$

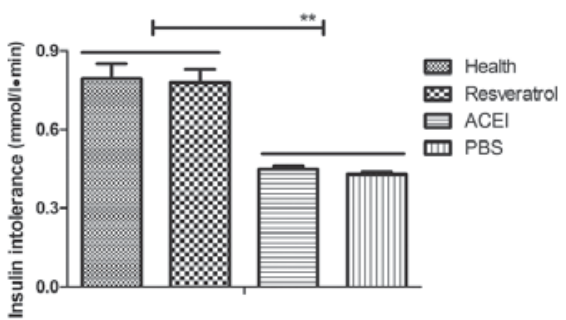

Figure 2. Benefits of resveratrol on glucose metabolism and insulin intolerance in mice with chronic renal failure. (A) Changes in body weight; (B) blood glucose concentration; (C) glucose intolerance; (D) absolute energy intake; (E) insulin levels; and (F) insulin tolerance. Mice with renal failure induced by type II diabetes were treated with resveratrol $(10 \mathrm{mg} / \mathrm{kg})$, the ACEI aldosterone $(10 \mathrm{mg} / \mathrm{kg})$ or the same volume of PBS, and healthy animals were used as controls. ${ }^{*} \mathrm{P}<0.05,{ }^{* *} \mathrm{P}<0.01$. ACEI, angiotensin-converting enzyme inhibitor.

A

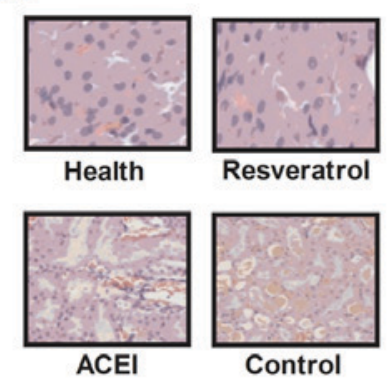

D

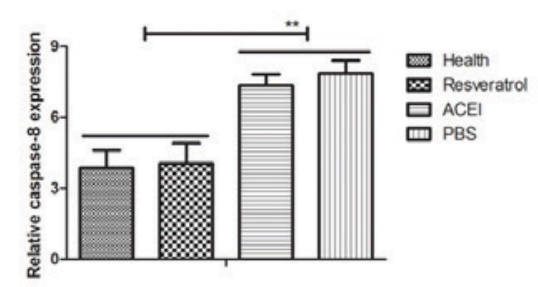

B

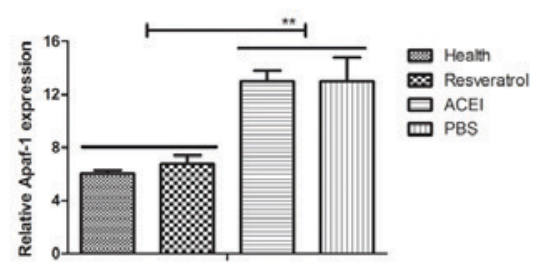

E

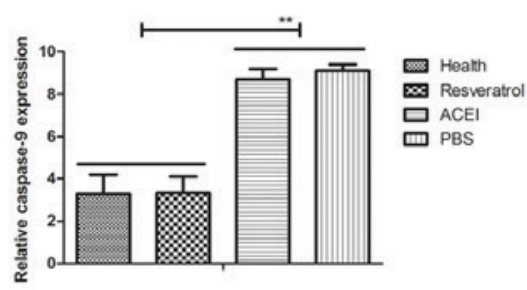

C

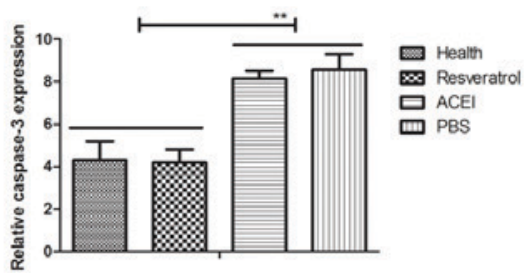

$\mathbf{F}$

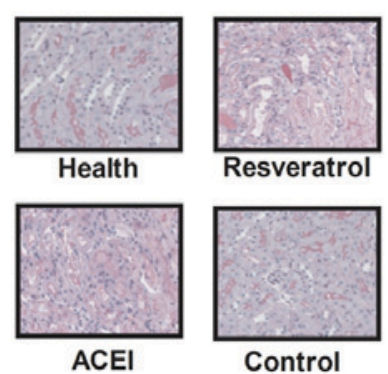

Figure 3. Analysis of the effect of resveratrol on apoptosis of renal cells from mice with chronic renal failure induced by type II diabetes. (A) Terminal deoxynucleotidyl transferase deoxyuridine triphosphate nick end labeling of apoptotic cells in renal tissues (magnification, x40). Levels of (B) Apaf-1, (C) cleaved caspase-3, (D) caspase-8 and (E) caspase-9 in renal cells from experimental mice after treatment with resveratrol, ACEI or PBS, as well as in healthy control mice. (F) Immunohistochemical analysis of caspase-3 expression in renal cells from experimental mice after treatment with resveratrol, ACEI or PBS, as well as in healthy control mice. ${ }^{* *} \mathrm{P}<0.01$. ACEI, angiotensin-converting enzyme inhibitor; Apaf-1, apoptotic protease activating factor 1 .

Resveratrol alters the expression and phosphorylation of AMPK levels in rats with chronic renal failure induced by type II diabetes. As AMPK is likely to be a target of resveratrol, via which it exerts its pharmacodynamic function, the AMPK signaling pathway in renal cells from experimental mice was examined. The expression and phosphorylation levels of AMPK were decreased after treatment with resveratrol (Fig. 4A and B). In addition, morphological observation revealed that resveratrol treatment improved the viability of renal cells determined by cells viability (Fig. 4C). Furthermore, immunostaining demonstrated that immunocyte numbers were decreased in renal cells of resveratrol-treated mice compared with those in the ACEI- and PBS-treated groups (Fig. 4D). AMPK activation was also decreased in renal cells of experimental mice after resveratrol treatment compared with that in the ACEI- and PBS-treated groups (Fig. 4E). In addition, 
A
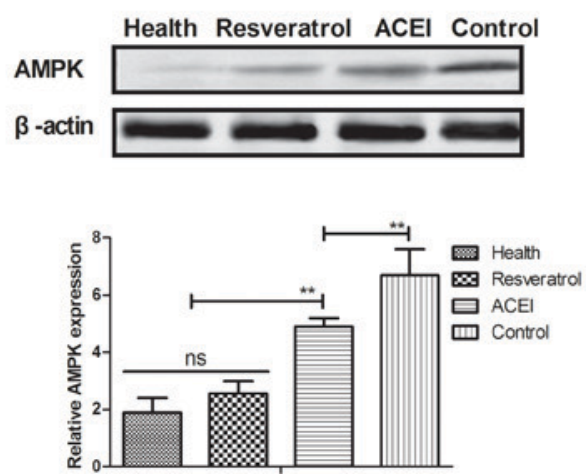

C

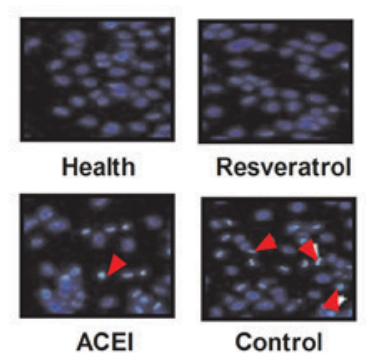

E

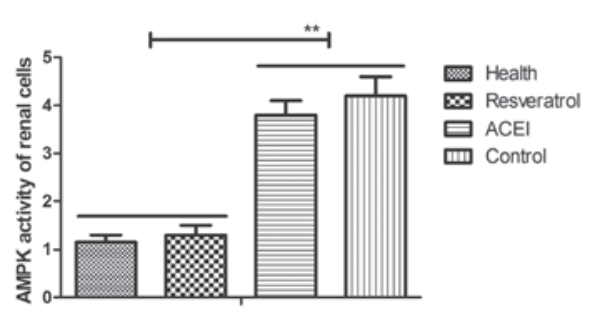

B
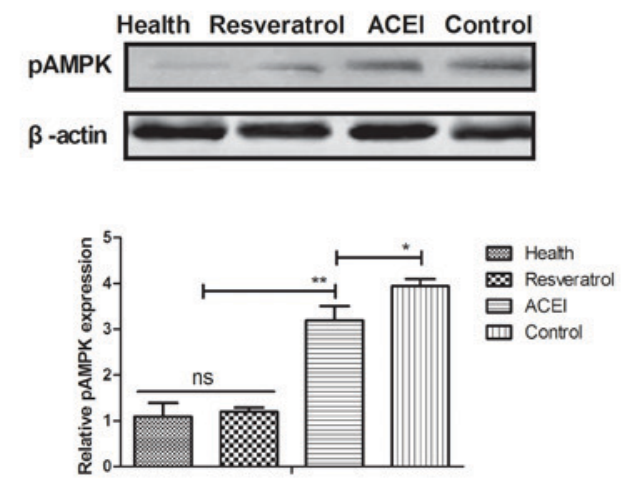

D
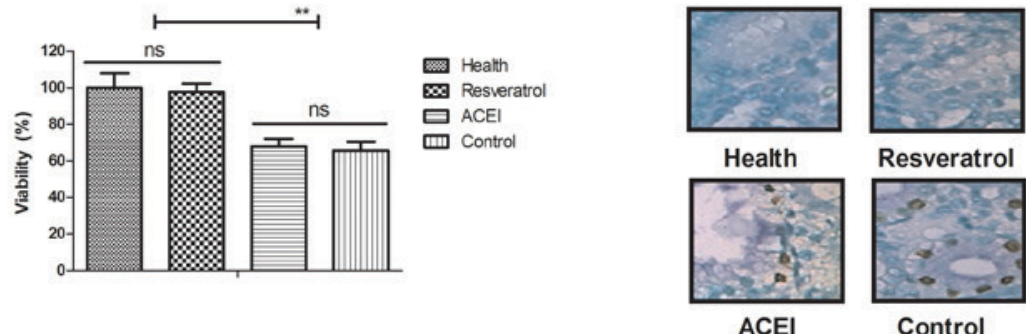

$\mathbf{F}$

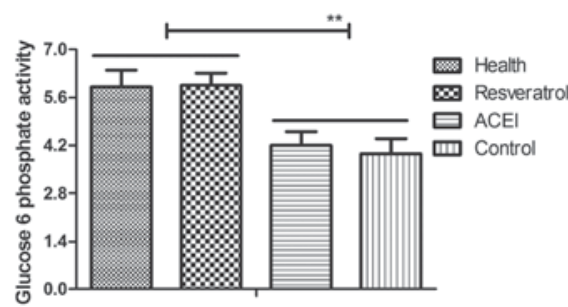

Figure 4. Effects of resveratrol on the expression and phosphorylation of AMPK in renal cells from mice with chronic renal failure. Analysis of (A) the expression and (B) phosphorylation levels of AMPK in renal cells. (C) Analysis of morphological changes in renal cells determined by cells viability. Arrows indicated atrophic cells (magnification, $\mathrm{x} 40$ ). (D) Glucose-6-phosphate activity in renal cells (magnification, $\mathrm{x} 40$ ). (E) Analysis of AMPK activity. (F) Glucose-6-phosphate activity in the serum. Mice with renal failure induced by type II diabetes were treated with resveratrol (10 mg/kg), the ACEI aldosterone $(10 \mathrm{mg} / \mathrm{kg})$ or the same volume of PBS, and healthy animals were used as controls. ${ }^{*} \mathrm{P}<0.05,{ }^{* * *} \mathrm{P}<0.01$. ACEI, angiotensin-converting enzyme inhibitor; pAMPK, phosphorylated adenosine monophosphate-activated protein kinase.

glucose-6-phosphate activity was significantly increased in the serum of experimental mice after treatment with resveratrol (Fig. 4F). These findings suggested that decreased AMPK activation by resveratrol contributes to the enzymatic capacity for glucose production in mice with chronic renal failure induced by type II diabetes, which may proceed via the AMPK-mediated signaling pathway.

Resveratrol decreases oxidative stress and anti-oxidant status in mice with chronic renal failure induced by type II diabetes. Oxidative stress contributes to type II diabetes-induced chronic renal failure, leading to increased apoptosis of renal cells in experimental mice. Hence, the present study investigated the oxidative stress and anti-oxidant status in renal cells after treatment with resveratrol. As presented in Fig. 5A, total thiol levels were decreased in renal cells after resveratrol treatment. Lipid peroxidation in renal cells of resveratrol-treated mice was increased compared with that in ACEI- and PBS-treated experimental mice (Fig. 5B). In addition, resveratrol treatment increased SOD activity and ROS levels in renal cells from experimental compared with that in ACEI- and PBS-treated experimental mice (Fig. 5C and D). In addition, increases of GSH in renal cells of experimental mice were found to be included in the benefits of resveratrol treatment (Fig. 5E). Furthermore, immunohistochemical detection of 4-hydroxy-2-nonenal (HNE) revealed that resveratrol treatment decreased the intense staining of HNE in renal cells compared with that in the controls, which may also indicate the enhancement of the anti-oxidant status (Fig. 5F). These results indicated that resveratrol treatment decreased oxidative stress and improved the anti-oxidant status in renal cells from mice with chronic renal failure induced by type II diabetes.

\section{Discussion}

Chronic renal failure is a syndrome resulting from serious metabolic disorders (18). Chronic renal failure affects nearly $8 \%$ of patients with type II diabetes and impairs the quality 
A

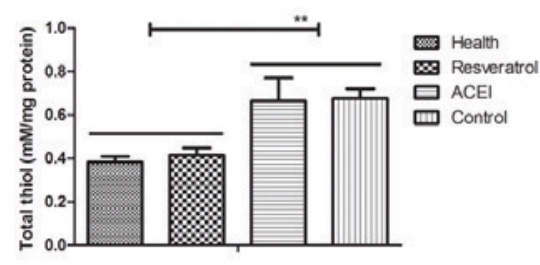

D

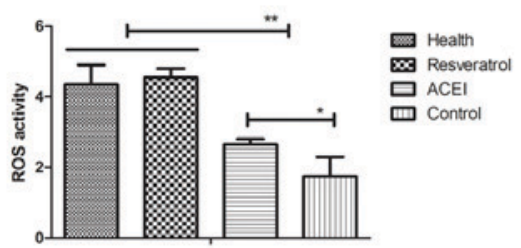

B

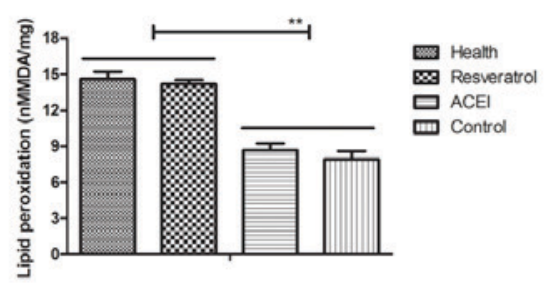

E

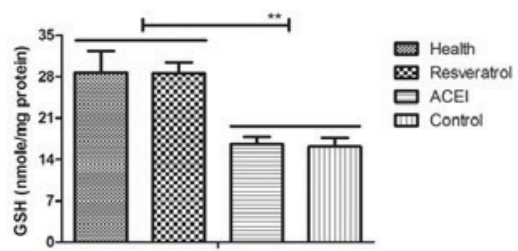

C
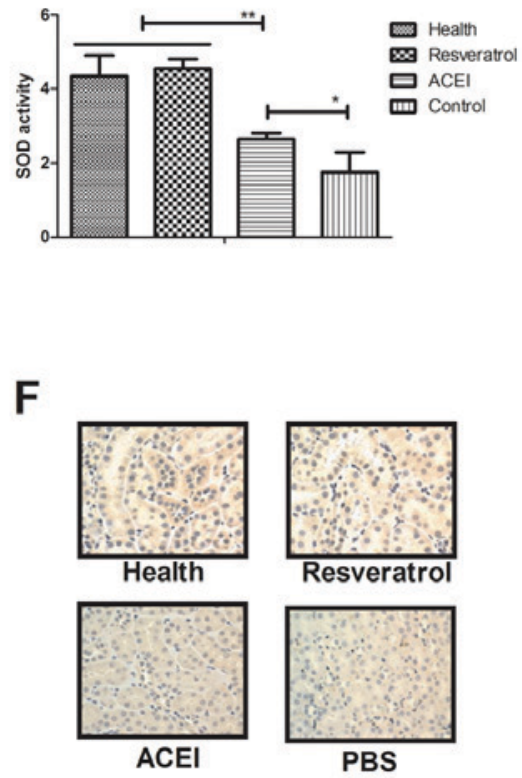

Figure 5. Regulatory effects of resveratrol on oxidative stress and anti-oxidant status in mice with chronic renal failure. (A) Total thiol levels in renal cells. (B) Lipid peroxidation in the experimental mice. (C) SOD activity, (D) ROS activity and (E) GSH levels in renal cells of experimental mice. (F) Immunohistochemical analysis of HNE in renal cells from experimental mice. Mice with renal failure induced by type II diabetes were treated with resveratrol (10 $\mathrm{mg} / \mathrm{kg}$ ), the ACEI aldosterone $(10 \mathrm{mg} / \mathrm{kg})$ or the same volume of PBS, and healthy animals were used as controls (magnification, $\mathrm{x} 40)$. ${ }^{*} \mathrm{P}<0.05,{ }^{* *} \mathrm{P}<0.01 . \mathrm{ACEI}$, angiotensin-converting enzyme inhibitor; SOD, superoxide dismutase; ROS, reactive oxygen species; GSH, glutathione; HNE, 4-hydroxy-2-nonenal.

of life of affected patients (34). Studies have indicated that type II diabetes frequently leads to chronic renal failure due to long-term insulin injection and disturbance of carbohydrate metabolism $(35,36)$. In addition, in patients with chronic renal failure induced by type II diabetes mellitus present insulinoma treatment with diazoxide is ineffective (37). Furthermore, type II diabetes-induced chronic renal failure leads to other clinical syndromes and increases mortality $(38,39)$. From these studies, it is concluded that type II diabetes may lead to chronic renal failure and result in other diseases, indicating the importance of the development of more efficient treatments for chronic renal failure induced by type II diabetes.

The present study investigated the therapeutic effects of resveratrol in a mouse model of chronic renal failure induced by type II diabetes and assessed the molecular mechanisms. Resveratrol is a naturally occurring polyphenol substance that has been proven to have multiple functions in the treatment of diabetes in various animal models $(40,41)$. However, the effect of resveratrol on chronic renal failure has remained to be fully elucidated. Resveratrol treatment was reported to restore peripheral insulin sensitivity in streptozotocin-induced or insulin receptor substrate 2-deficient diabetic mouse models (42). The present study found that insulin resistance, glucose metabolism and body weight in mice with chronic renal failure induced by type II diabetes were significantly improved after resveratrol treatment, which confirms the outcomes reported by previous studies $(43,44)$.

Inflammation is one of the most common characteristics of patients with chronic renal failure (45). In addition, oxidative stress is associated with the progression of patients with chronic renal failure and metabolic syndrome (46). Oxidative stress is also associated with neutrophil function in cats with chronic renal failure (47). A previous study found that resveratrol treatment mitigates hepatic injury, oxidative stress and apoptosis in a rat model by regulating nuclear factor (NF) $\kappa$ B signaling pathway (48). Resveratrol also protects cells against titanium particle-induced aseptic loosening through reduction of oxidative stress and inactivation of $\mathrm{NF}-\kappa \mathrm{B}$ via inhibition of nitric oxide production, ROS generation and lipid peroxidation (49). The present study indicated that resveratrol treatment not only decreased the apoptosis of renal cells, but also markedly improved oxidative stress of renal cells isolated from experimental mice. Furthermore, cytokines, including IL-6, IL-17, IL-1 $\beta$, TNF- $\alpha$, IL10 and VEGF, were assessed in the sera of mice with chronic renal failure induced by type II diabetes. The results demonstrated that after resveratrol treatment, the decreases of IL- 6 and VEGF as well as the increases of IL-17, IL-1 $\beta$, TNF- $\alpha$ and IL-10 in the experimental vs. control mice were inhibited by resveratrol. Furthermore, the AMPK signaling pathway was found to be involved in the resveratrol-mediated improvement of the chronic renal failure induced by type II diabetes.

The AMPK signaling pathway has an important role in the function of renal cells (50). A previous study reported that resveratrol attenuated TNF- $\alpha$-induced matrix metalloproteinase- 3 expression in human nucleus pulposus cells by activating the AMPK/Sirtuin1 signaling pathway (51). In addition, resveratrol enhances the anti-tumor effects of temozolomide in glioblastoma via a ROS-dependent AMPK/tuberous sclerosis $1 /$ mammalian target of rapamycin signaling pathway (52). Furthermore, a previous study suggested that resveratrol induces apoptosis in chemoresistant cancer cells via modulation of the AMPK signaling pathway (53). These results suggested that AMPK may be involved in apoptosis and oxidative stress 
in renal cells. Importantly, the present results revealed that resveratrol treatment regulated apoptosis and oxidative stress via the AMPK signaling pathway. The decreased activity of AMPK contributes to the recovery of renal cells isolated from mice with chronic renal failure induced by type II diabetes. The findings of the present study indicated that resveratrol treatment improves glucose metabolism and insulin resistance via activation of glucose-6-phosphate through the AMPK signaling pathway. Interestingly, resveratrol treatment not only improved the glucose levels and lipid profile, but also maintained glucose homeostasis and lipid metabolism in mice with chronic renal failure. Of note, apoptosis and oxidative stress in renal cells were inhibited by the treatment with resveratrol, which also contributed to the increased body weight and insulin intolerance.

In conclusion, the results of the present study expanded on the known molecular mechanism of action of resveratrol in the treatment of mice with chronic renal failure induced by type II diabetes. Resveratrol treatment markedly improved glucose metabolism and insulin resistance in mice with chronic renal failure induced by type II diabetes. The results indicated that resveratrol treatment targeting AMPK signaling may be a potential strategy to protect against apoptosis and oxidative stress in renal cells. Taken together, regulation of the AMPK pathway by resveratrol may serve as an effective treatment for chronic renal failure, which also suggests the therapeutic potential of other compounds modulating the AMPK signaling pathway.

\section{Acknowledgements}

The authors would like to thank Dr Zhi Li (Department of Sports Medicine, North University of China) for assisting in performing the statistical analysis.

\section{Funding}

No funding was received.

\section{Availability of data and materials}

The analyzed data sets generated during the study are available from the corresponding author on reasonable request.

\section{Authors' contributions}

LZ performed the experiments. HG analyzed and designed experiments for the present study.

\section{Ethics approval and consent to participate}

This pre-clinical study was approved by the Ethics Committee of Fenyang College Shanxi Medical University (Fenyang, China).

\section{Consent for publication}

Not applicable.

\section{Competing interests}

The authors declare that they have no competing interests.

\section{References}

1. Ibrahim NE, Gaggin HK, Rabideau DJ, Gandhi PU, Mallick A and Januzzi JL Jr: Worsening renal function during management for chronic heart failure with reduced ejection fraction: Results from the Pro-BNP outpatient tailored chronic heart failure therapy (PROTECT) study. J Card Fail 23: 121-130, 2016.

2. Sati A, Jha A, Moulick PS, Shankar S, Gupta S, Khan MA, Dogra $M$ and Sangwan VS: Corneal endothelial alterations in chronic renal failure. Cornea 35: 1320-1330, 2016.

3. Iwamuro M, Kanzaki H, Tanaka T, Kawano S, Kawahara Y and Okada H: Lanthanum phosphate deposition in the gastric mucosa of patients with chronic renal failure. Nihon Shokakibyo Gakkai Zasshi 113: 1216-1222, 2016 (In Japanese).

4. Jain P, Kanagal-Shamanna R, Wierda W, Ferrajoli A, Keating M and Jain N: Membranoproliferative glomerulonephritis and acute renal failure in a patient with chronic lymphocytic leukemia: Response to obinutuzumab. Hematol Oncol Stem Cell Ther 10: 151-154, 2017.

5. Nakano S, Masuda K, Asanuma T and Nakatani S: The effect of chronic renal failure on cardiac function: An experimental study with a rat model. J Echocardiogr 14: 156-162, 2016.

6. Sathyanarayana V, Patel MT, Raghavan S and Naresh D Simultaneous bilateral femur neck fracture in a young adult with chronic renal failure-a case report and review of literature. J Orthop Case Reports 5: 24-26, 2015.

7. Malekmakan L, Malekmakan A, Daneshian A, Pakfetrat M and Roosbeh J: Hypertension and diabetes remain the main causes of chronic renal failure in Fars Province, Iran 2013. Saudi J Kidney Dis Transpl 27: 423-424, 2016.

8. Mao W, Zhang L, Zou C, Li C, Wu Y, Su G, Guo X, Wu Y, Lu F, Lin $\mathrm{Q}$, et al: Rationale and design of the helping ease renal failure with bupi yishen compared with the angiotensin II antagonist losartan (HERBAAL) trial: A randomized controlled trial in non-diabetes stage 4 chronic kidney disease. BMC Complement Altern Med 15: 316, 2015.

9. Takase H, Nakazawa A, Yamashita S, Toriyama T, Sato K, Ueda R and Dohi Y: Pioglitazone produces rapid and persistent reduction of vascular inflammation in patients with hypertension and type 2 diabetes mellitus who are receiving angiotensin II receptor blockers. Metabolism 56: 559-564, 2007.

10. Varughese GI and Lip GY: Hypertension in patients with type-II diabetes: Relation to urinary albumin excretion, endothelial function and inflammation. J Hum Hypertens 19: 421-424, 2005.

11. Yamamoto S, Okada Y, Mori H, Nishida K, Uriu K and Tanaka Y: Type 2 diabetes mellitus complicated by hypertension in Japanese patients: Switching treatment from high-dose angiotensin II receptor blockers to losartan plus hydrochlorothiazide. Intern Med 53: 1283-1289, 2014.

12. Kalaitzidis R and Bakris G: Management of hypertension in patients with diabetes: The place of angiotensin-II receptor blockers. Diabetes Obes Metab 11: 757-769, 2009.

13. Hasvold LP, Bodegård $\mathrm{J}$, Thuresson M, Stålhammar J, Hammar N, Sundström J, Russell D and Kjeldsen SE: Diabetes and CVD risk during angiotensin-converting enzyme inhibitor or angiotensin II receptor blocker treatment in hypertension: A study of 15,990 patients. J Hum Hypertens 28: 663-669, 2014.

14. Daimon M, Kamba A, Murakami H, Takahashi K, Otaka H, Makita K, Yanagimachi M, Terui K, Kageyama K, Nigawara $\mathrm{T}$, et al: Association between pituitary-adrenal axis dominance over the renin-angiotensin-aldosterone system and hypertension. J Clin Endocrinol Metab 101: 889-897, 2016.

15. Ficek J, Malyszko J and Chudek J: Renalase and its role in the development of hypertension in patients with chronic renal failure. Przegl Lek 72: 306-308, 2015 (In Polish).

16. Finlay E: Review: Most interventions for preventing bone disease in chronic renal failure improved biochemical outcomes. Arch Dis Child Edu Pract Ed 97: 40, 2012.

17. Kasacka I: Review article-involvement of gastric APUD cells in chronic renal failure. Acta Histochem 105: 319-327, 2003.

18. Bos-Touwen I, Schuurmans M, Monninkhof EM, Korpershoek Y, Spruit-Bentvelzen L, Ertugrul-van der Graaf I, de Wit N and Trappenburg J: Patient and disease characteristics associated with activation for self-management in patients with diabetes, chronic obstructive pulmonary disease, chronic heart failure and chronic renal disease: A cross-sectional survey study. PloS One 10: $\mathrm{e} 0126400,2015$. 
19. Nair PA, Jivani NB and Diwan NG: Kyrle's disease in a patient of diabetes mellitus and chronic renal failure on dialysis. J Family Med Prim Care 4: 284-286, 2015.

20. Evans HM, Howe PR and Wong RH: Clinical evaluation of effects of chronic resveratrol supplementation on cerebrovascular function, cognition, mood, physical function and general well-being in postmenopausal women-rationale and study design. Nutrients 8: 150,2016.

21. Toth P, Tarantini S, Tucsek Z, Ashpole NM, Sosnowska D, Gautam T, Ballabh P, Koller A, Sonntag WE, Csiszar A and Ungvari Z: Resveratrol treatment rescues neurovascular coupling in aged mice: Role of improved cerebromicrovascular endothelial function and downregulation of NADPH oxidase. Am J Physiol Heart Circ Physiol 306: H299-H308, 2014.

22. Beaudoin MS, Snook LA, Arkell AM, Simpson JA, Holloway GP and Wright DC: Resveratrol supplementation improves white adipose tissue function in a depot-specific manner in Zucker diabetic fatty rats. Am J Physiol Regul Integr Comp Physiol 305 R542-R551, 2013.

23. González-Rodríguez Á, Santamaría B, Mas-Gutierrez JA, Rada P, Fernández-Millán E, Pardo V, Álvarez C, Cuadrado A, Ros M, Serrano M and Valverde ÁM: Resveratrol treatment restores peripheral insulin sensitivity in diabetic mice in a sirt1-independent manner. Mol Nutr Food Res 59: 1431-1442, 2015.

24. Gencoglu H, Tuzcu M, Hayirli A and Sahin K: Protective effects of resveratrol against streptozotocin-induced diabetes in rats by modulation of visfatin/sirtuin-1 pathway and glucose transporters. Int J Food Sci Nutr 66: 314-320, 2015.

25. Carrizzo A, Puca A, Damato A, Marino M, Franco E, Pompeo F, Traficante A, Civitillo F, Santini L, Trimarco V and Vecchione C: Resveratrol improves vascular function in patients with hypertension and dyslipidemia by modulating NO metabolism. Hypertension 62: 359-366, 2013.

26. Gulvady AA, Ciolino HP, Cabrera RM and Jolly CA: Resveratrol inhibits the deleterious effects of diet-induced obesity on thymic function. J Nutr Biochem 24: 1625-1633, 2013.

27. Guan Y, Cui ZJ, Sun B, Han LP, Li CJ and Chen LM: Celastrol attenuates oxidative stress in the skeletal muscle of diabetic rats by regulating the AMPK-PGC1 $\alpha$-SIRT3 signaling pathway. Int J Mol Med 37: 1229-1238, 2016.

28. Madsen A, Bjune JI, Bjorkhaug L, Mellgren G and Sagen JV: The cAMP-dependent protein kinase downregulates glucose-6-phosphatase expression through ROR $\alpha$ and SRC-2 coactivator transcriptional activity. Mol Cell Endocrinol 419: 92-101, 2016

29. Xu S, Lv Y, Zhao J, Wang J, Zhao X and Wang S: Inhibitory effects of Shenkang injection and its main component emodin on the proliferation of high glucoseinduced renal mesangial cells through cell cycle regulation and induction of apoptosis. Mol Med Rep 14: 3381-3388, 2016.

30. He Y, Chen W, Hu Y, Luo B, Wu L, Qiao Y, Mo Q, Xu R, Zhou Y, Ren Z, et al: E. adenophorum induces cell cycle and apoptosis of renal cells through mitochondrial pathway and caspase activation in saanen goat. PloS One 10: e0138504, 2015.

31. Livak KJ and Schmittgen TD: Analysis of relative gene expression data using real-time quantitative PCR and the 2(-Delta Delta C(T)) method. Methods 25: 402-408, 2001.

32. Santos SS, Carmo AM, Brunialti MK, Machado FR, Azevedo LC, Assunção M, Trevelin SC, Cunha FQ and Salomao R: Modulation of monocytes in septic patients: Preserved phagocytic activity, increased ROS and NO generation, and decreased production of inflammatory cytokines. Intensive Care Med Exp 4: 5, 2016.

33. Hyde GD, Taylor RF, Ashton N, Borland SJ, Wu HS, Gilmore AP and Canfield AE: Axl tyrosine kinase protects against tubulo-interstitial apoptosis and progression of renal failure in a murine model of chronic kidney disease and hyperphosphataemia. PloS One 9: e102096, 2014.

34. Alba A, Morales J, Ferrario M, Zehnder C, Aguiló J, Zavala C, Herzog C, Calabran L, Contreras L, Espinoza R, et al Simultaneous kidney and pancreas transplantation (SKPT) in patients with type 1 diabetes and chronic renal failure: Experience in 12 patients in Chile. Rev Med Chil 139: 11-18, 2011 (In Spanish).

35. Wittmann I, Molnár GA, Wagner L, Köszegi T, Wagner Z, Laczy B, Tamaskó M, Markó L, Mohás M and Nagy J: Single dose of acetylsalicylic acid in patients with Type 2 diabetes mellitus and/or chronic renal failure ameliorates anaemia by decreasing the rate of neocytolysis. Acta Physiol Hung 94: $159-166,2007$
36. Shamaeva EN, Shestakova MV, Kim IG, Stoliarevich ES and Tomilina NA: Transplantation of the kidney-optimal treatment of patients suffering from diabetes mellitus type 1 with terminal chronic renal failure. Ter Arkh 79: 40-44, 2007 (In Russian).

37. Shimizu M, Suzuki K, Tsuchida K, Kojima M, Hiraishi H and Aso Y: Insulinoma in a patient with chronic renal failure due to type 2 diabetes mellitus treated effectively with diazoxide. Intern Med 54: 621-625, 2015.

38. Miyazato J,Horio T, Takiuchi S, Kamide K, Sasaki O, Nakamura S, Nakahama H, Inenaga T, Takishita S and Kawano Y: Left ventricular diastolic dysfunction in patients with chronic renal failure: Impact of diabetes mellitus. Diabet Med 22: 730-736, 2005.

39. Majdan M, Kurowska M, Orlowska-Kowalik G and Drop A: Ultrasonographic evaluation of kidneys in type-2 diabetes patients without overt nephropathy and with chronic renal failure. Wiad Lek 58: 25-28, 2005 (In Polish).

40. Ding DF, You N, Wu XM, Xu JR, Hu AP, Ye XL, Zhu Q, Jiang XQ, Miao H, Liu C and Lu YB: Resveratrol attenuates renal hypertrophy in early-stage diabetes by activating AMPK. Am J Nephrol 31: 363-374, 2010.

41. Szkudelska K and Szkudelski T: Resveratrol, obesity and diabetes. Eur J Pharmacol 635: 1-8, 2010.

42. Thirunavukkarasu M, Penumathsa SV, Koneru S, Juhasz B, Zhan L, Otani H, Bagchi D, Das DK and Maulik N: Resveratrol alleviates cardiac dysfunction in streptozotocin-induced diabetes: Role of nitric oxide, thioredoxin, and heme oxygenase. Free Radic Biol Med 43: 720-729, 2007.

43. Ungvari Z and Csiszar A: Resveratrol confers endothelial protection in insulin-dependent diabetes mellitus: Editorial to: 'Resveratrol shows vasoprotective effect reducing oxidative stress without affecting metabolic disturbances in insulin-dependent diabetes of rabbits' by F. Akar et al. Cardiovasc Drugs Ther 25: 111-113, 2011.

44. Huang JP, Huang SS, Deng JY, Chang CC, Day YJ and Hung LM: Insulin and resveratrol act synergistically, preventing cardiac dysfunction in diabetes, but the advantage of resveratrol in diabetics with acute heart attack is antagonized by insulin. Free Radic Biol Med 49: 1710-1721, 2010.

45. Verheul MK, van Erp SJ, van der Woude D, Levarht EW, Mallat MJ, Verspaget HW, Stolk J, Toes RE, van der Meulen-de Jong AE, Hiemstra PS, et al: Anti-carbamylated protein antibodies: A specific hallmark for rheumatoid arthritis. Comparison to conditions known for enhanced carbamylation; renal failure, smoking and chronic inflammation. Ann Rheum Dis 75: 1575-1576, 2016.

46. Medvedeva E, Berezin I, Surkova E, Yaranov D and Shchukin Y: Galectin-3 in patients with chronic heart failure: Association with oxidative stress, inflammation, renal dysfunction and prognosis. Minerva Cardioangiol 64: 595-602, 2016.

47. Keegan RF and Webb CB: Oxidative stress and neutrophil function in cats with chronic renal failure. J Vet Intern Med 24: 514-519, 2010.

48. Seif El-Din SH, El-Lakkany NM, Salem MB, Hammam OA, Saleh S and Botros SS: Resveratrol mitigates hepatic injury in rats by regulating oxidative stress, nuclear factor-kappa $\mathrm{B}$, and apoptosis. J Adv Pharm Technol Res 7: 99-104, 2016

49. Luo G, Li Z, Wang Y, Wang H, Zhang Z, Chen W, Zhang Y, Xiao Y, $\mathrm{Li} \mathrm{C}$, Guo Y and Sheng P: Resveratrol protects against titanium particle-induced aseptic loosening through reduction of oxidative stress and inactivation of NF-кB. Inflammation 39: 775-785, 2016.

50. Lieberthal W, Tang M, Lusco M, Abate M and Levine JS: Preconditioning mice with activators of AMPK ameliorates ischemic acute kidney injury in vivo. Am J Physiol Renal Physiol 311: F731-F739, 2016.

51. Wang XH, Zhu L, Hong X, Wang YT, Wang F, Bao JP, Xie XH, Liu L and Wu XT: Resveratrol attenuated TNF- $\alpha$-induced MMP-3 expression in human nucleus pulposus cells by activating autophagy via AMPK/SIRT1 signaling pathway. Exp Biol Med (Maywood) 241: 848-853, 2016.

52. Yuan Y, Xue X, Guo RB, Sun XL and Hu G: Resveratrol enhances the antitumor effects of temozolomide in glioblastoma via ROS-dependent AMPK-TSC-mTOR signaling pathway. CNS Neurosci Ther 18: 536-546, 2012.

53. Hwang JT, Kwak DW, Lin SK, Kim HM, Kim YM and Park OJ: Resveratrol induces apoptosis in chemoresistant cancer cells via modulation of AMPK signaling pathway. Ann N Y Acad Sci 1095: 441-448, 2007.

This work is licensed under a Creative Commons Attribution-NonCommercial-NoDerivatives 4.0 International (CC BY-NC-ND 4.0) License. 